\title{
Multislice CT in adult colocolic intussusception: case report and review of the literature
}

\author{
Frank Floemer • Heidi Bissig • Daniel Oertli • \\ Georg Bongartz $\cdot$ Christian T. Hamel
}

Received: 15 October 2007 / Accepted: 22 October 2007 / Published online: 15 November 2007

(C) Am Soc Emergency Radiol 2007

\begin{abstract}
Intussusception in adults is generally a rare diagnosis and generally different from intussusception in children in terms of clinical presentation, etiology, and incidence (Begos et al., Am J Surg, 173:88-94, 1997; Watson and Bisset, Clin Radiol, 49:723-726, 1994; Felix et al., Am J Surg, 131:723-726, 1976). One third of these affect the large bowel. Adult intussusception shows clinically uncharacteristic symptoms of bowel obstruction; thus, the diagnosis is often clinically missed. We report the case of a 39-year-old woman suffering from long-term abdominal pain. This case report discusses the clinical advantages of multislice computed tomography for the diagnosis of adult intussusception and shows a comprehensive overview of the literature.
\end{abstract}

Keywords Colon · Intussusception - Colon intussusception · Computed tomography $(\mathrm{CT}) \cdot$ Multidetector row computed tomography (MDCT)

F. Floemer $(\bowtie) \cdot$ G. Bongartz

Department of Radiology, University Hospital Basel, Petersgraben 4,

4031 Basel, Switzerland

e-mail: frank.floemer@t-online.de

D. Oertli $\cdot$ C. T. Hamel

Department of Surgery, University Hospital Basel,

Petersgraben 4,

4031 Basel, Switzerland

H. Bissig

Department of Pathology, University Hospital Basel,

Schoenbeinstrasse 4,

4003 Basel, Switzerland

\section{Introduction}

The term intussusception defines the condition that a proximal bowel segment (intussusceptum) folds into an adjacent distal segment (intussuscipiens). Intussusception in adults is completely different from children in terms of clinical presentation, etiology, and incidence [1-4]. The condition is relatively uncommon in adults [5] and is estimated to be responsible for $1 \%$ of all bowel obstructions [6] and only $0.003-0.02 \%$ of all hospital admissions [4]. About one third of all adult intussusceptions are colonic, and the remaining two thirds mainly affect the small bowel. Underlying intraluminal pathology is found in about 80 $90 \%$ of patients. The pathomechanism is thought to be altered bowel peristalsis at the intraluminal lesion, which is then a lead point for the intussusceptum $[4,5]$.

Intussusception is classified as enteric, ileocolic, ileocecal, or colonic, depending on the location of the condition. Enteric lesions are restricted to the small intestine. Prolapse of the ileum through the ileocecal valve is called ileocolic intussusception. In ileocecal intussusception, the ileocecal valve is the lead point. Colonic intussusception is strictly confined to the large intestine [7]. Adult intussusception tends to develop at intersections between freely moving bowel segments and segments that are either retroperitoneal or adhesionally fixed [1].

Clinical symptoms of intussusception are typical for bowel obstruction and allow a wide range of differential diagnoses. For this reason, intussusception can easily be missed. In this context, the present article describes the multislice computed tomography (MSCT) imaging characteristics of adult colocolic intussusception, which are pathognomonic for the condition. The diagnostic potential of multislice CT and the impact of imaging findings for 
therapeutic planning will be discussed on the basis of a literature review.

\section{Case report}

A 39-year-old woman with a 3-day history of malaise and worsening sharp abdominal pain in the left upper quadrant after food intake was referred by her family doctor. She reported repeated abdominal cramps and nausea after meals, starting 3 months before admission. The patient also suffered from progressive diarrhea with the stools congested by mucous without blood. She reported no fever or chills but lost $3 \mathrm{~kg}$ of body weight during this period. White blood cell count was $8.5 \times 10^{9} / 1$, hemoglobin level was $126 \mathrm{~g} / \mathrm{l}$, and hematocrit $38 \%$. Concentrations of C-reactive protein (CRP) and of electrolytes were normal. On physical examination, diffuse upper abdominal tenderness and a firm and tender mass in the epigastric area were found. The patient was clinically stable. MSCT was performed to provide more information about the extent, origin, and possible malignancy of the abdominal mass.

MSCT of the abdomen and pelvis was performed with a Sensation 16 slice scanner (Siemens Medical Systems, Iselin, USA); $30 \mathrm{ml}$ of Telebrix ${ }^{\circledR}$ Gastro (ioxithalamate) were diluted in $1,000 \mathrm{ml}$ of water and orally administered $1 \mathrm{~h}$ before imaging. Throughout image acquisition, the patient was in a supine position. Automatic bolus tracking was used for image acquisition of the portal venous phase after i.v. application of $100 \mathrm{ml}$ iodine contrast medium. Imaging was obtained using a helical mode with a slice collimation of $16 \times 1.5 \mathrm{~mm} / \mathrm{s}, 360^{\circ}$ rotation time of $1 \mathrm{~s}$, $24 \mathrm{~mm} / \mathrm{s}$ table speed, and $10 \mathrm{~s}$ scan time. A total of 260 images were acquired. Diagnostic work-up of the CT images was performed on a Leonardo computer workstation (Siemens Medical Systems).

Combined oral and i.v. contrast enhanced MSCT showed an extensive lesion, measuring $>12 \mathrm{~cm}$ length. Invagination started in the right colic flexure (Fig. 1a). In the center of the lesion, a compressed canal of soft tissue density with
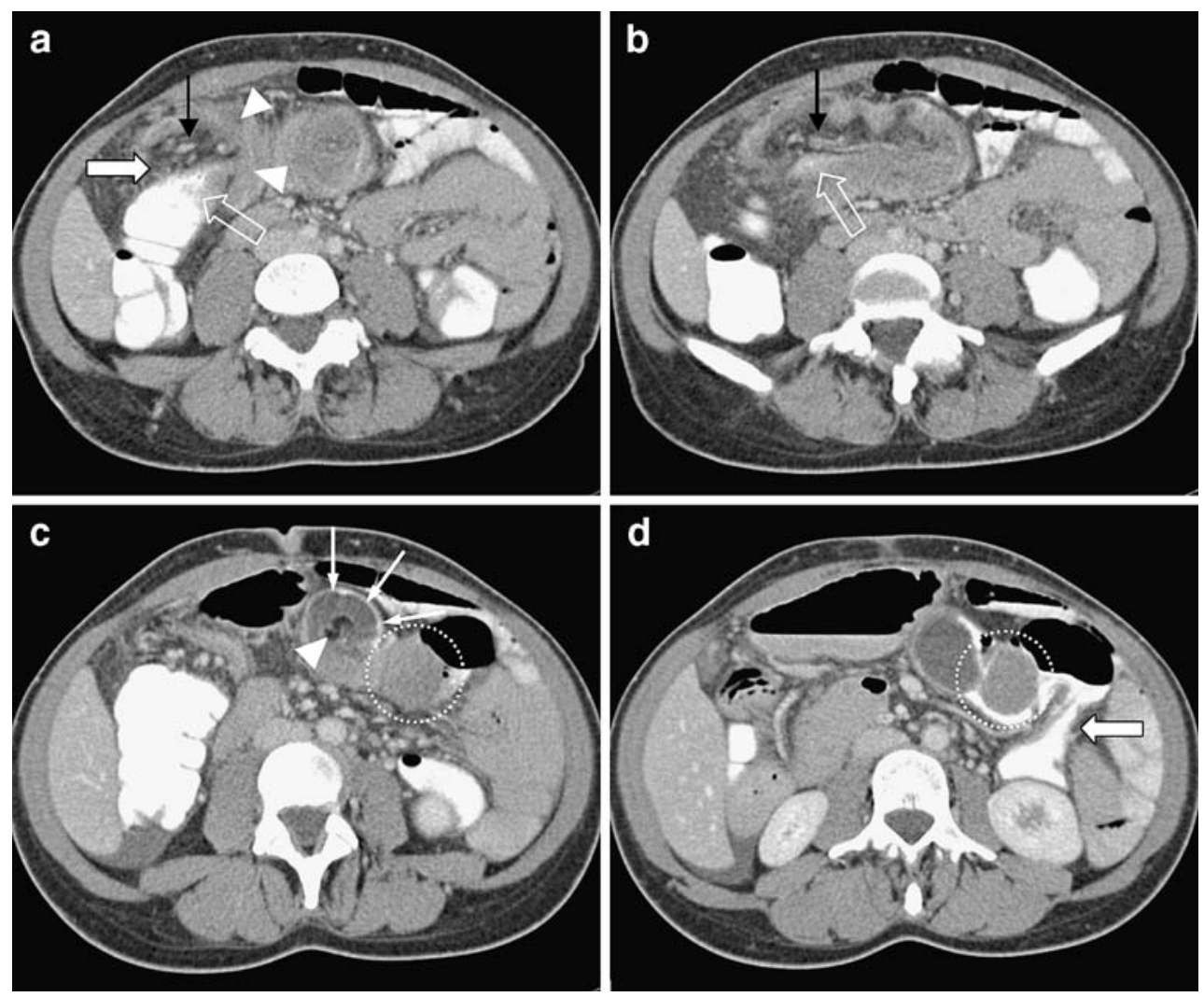

Fig. 1 Axial CT images of the proximal colon segment (intussusceptum) infolded with mesenteric fat (fatty dense tissue) and vessels (interposed stripes) into the distal colon segment (intussuscipient) along the transverse colon. a Starting point of the intussusceptum (white transparent arrow) with mesenteric fat (white arrow) and vessels (black arrow) into the intussuscipient (white arrowheads). b Intraluminal contrast media filled intussusceptum (white transparent arrow) folding into the intussuscipient. Stripes within the mesenteric

fat represent the mesenteric blood vessels (black arrows). c Mesenteric fat shown as fatty dense center (white arrowhead) and the contrast media-filled compressed lumen of the intussuscipient (white arrows) are depicted in the further continuation of the lesion. Furthermore, the intraluminal neoplasia is seen (circle). d The pedunculated mass is surrounded by contrast media (circle). Incomplete obstruction can be diagnosed by contrast media filled lumen of the descending colon (large white arrow) 
intraluminal contrast medium (Figs. $1 \mathrm{~b}$ and $2 \mathrm{~b}$ ) was seen, surrounded by fatty tissue with interposed stripes (Fig. 1b). The intraluminal fatty tissue was bordered by a sheath of peripheral hyperdense soft tissue. The compressed lumen of the distal bowel segment was depicted as a ring like structure filled with contrast media surrounding the mesenteric intraluminal fat (Fig. 1c). On the short axis, the lesion was target shaped, with a maximum diameter of $4.5 \mathrm{~cm}$ (Figs. 1c and 2a). Near the left colic flexure, a welldemarcated pedunculated intraluminal mass of $3.5 \times 3.0 \times$ $3.7 \mathrm{~cm}$ diameter and homogenous contrast enhancement was found (Figs. 1d and 2b). The obstruction was incomplete proven by the contrast medium-filled bowel lumen distal to the lead lesion (Fig. 1d). Bowel wall thickening and small bowel distension were excluded. Infection, intra-abdominal fluid accumulation, ischemia, free intra-abdominal air, and metastatic tumor spread were absent. The macroscopic layered colocolic "bowel-withinbowel" appearance led to the diagnosis of colocolic intussusception along the transverse colon, derived from an intraluminal neoplastic lead lesion (Figs. 1c,d and $2 b-d)$.

It was therefore decided to perform an emergency operation. Colonoscopic reduction was not done because of the large size of the tumor to be operated to avoid the risk of perforation.
Surgery revealed mild distension of the proximal colon and mild inflammatory changes of the transverse colon. In the vicinity of these changes, an intraluminal mass of $4 \mathrm{~cm}$ was palpated. Probably as a result of the general anesthesia and complete muscular relaxation, invagination was resolved at the time of open surgery. Formal resection of the transverse colon with adequate margins was performed. The resected and opened specimen showed a 4-cm polypous lesion (Fig. 2c). Histopatholoy revealed a tubulovillous adenomatous polyp with high grade dysplasia and superficial necrosis (Fig. 2d). The patient did not suffer from any postoperative complications and left the hospital on day 6 after the operation. Colonoscopy 2 months after the surgery excluded metachronic disease.

\section{Discussion}

A majority of the intussusceptions (85-95\%) have been described in children and are idiopathic. About 5-15\% appear in adults $[3,8]$. In $90 \%$ of adult intussusceptions, an underlying lesion can be identified [1]. Although the pathomechanism of idiopathic intussusception remains unclear, when there is an intraluminal bowel lesion, a leading edge is present that is pushed forward by peristalsis into the distal bowel segment $[1,4,5,7,9]$.
Fig. 2 Multiplanar CT images and histopathology characteristics of the intraluminal lead lesion. a Sagittal plane reveals the target sign (white arrows). b The coronar plane shows the whole extension of the contrast media-filled intussusceptum (large white arrow) and the pedunculated lead lesion (circle). c Surgical specimen demonstrates the solid pedunculated mass lodged in the proximal part of the transverse colon. d Whole-mount section of the pedunculated tubulovillous adenoma with a mixture of both tubular and villous growth patterns and focal high-grade dysplasia. (hematoxylin and eosin)
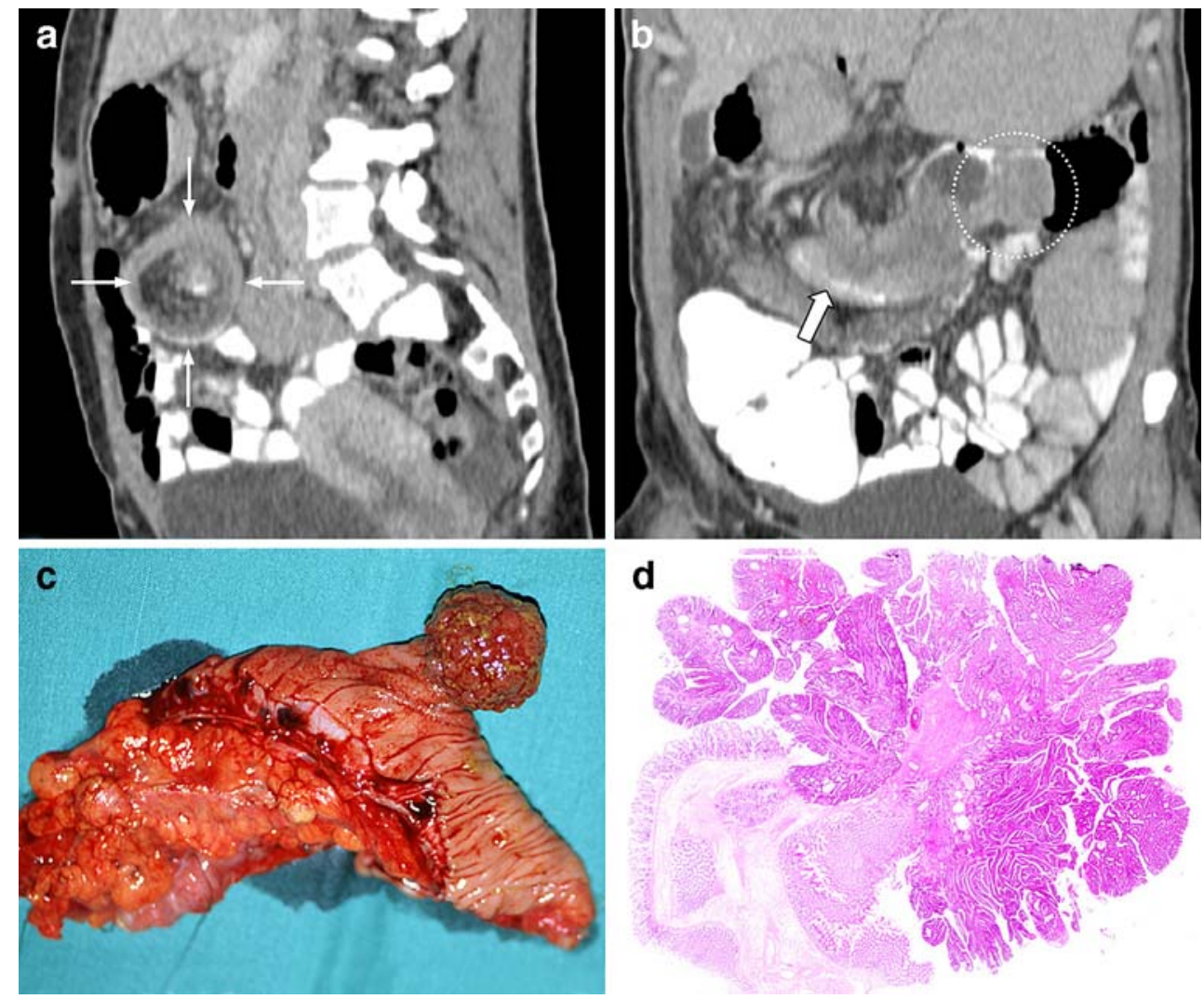
Leading symptoms in adult intussusception are those of mechanical bowel obstruction, predominantly nausea, emesis, and abdominal pain (73-78\%) [1, 7]. Palpable abdominal masses are found in $10-20 \%$ of patients depending on their location and dimension [4, 7]. Prolonged pain from 2 months -5 years has been reported with the combination of benign lead lesion and enteroenteric intussusception. The combination of malignancy and colocolic intussusception tends to cause shorter lasting pain periods $[1,7]$. Long standing, clinically painless intussusception has been found in patients with tuberculosis [10]. However, the duration and presence of clinical symptoms are not reliable for clinical diagnosis [7]. The clinical diagnosis of intussusception in adults is frequently missed [4]. Only about $32-38 \%$ of cases are clinically correct diagnosed [1]. Hence, further research on the outcomes based on modern imaging techniques is of clinical interest [11].

Angiographic criteria for intestinal intussusception have been described, including abrupt disappearance of mesenteric vessels, angulation of invaginated vessels, overlap of mesentery vessels by branches of the intussuscipiens, and abrupt changes of the vasa rectae of the intussuscipiens [12]. However, angiography is an unnecessarily invasive procedure.

Positron emission computed tomography has been demonstrated to be helpful in patients with intussusception and adenocarcinoma of the colon and showed a nonspecific fluordeoxyglucose uptake by the lead lesion [13]. Radionucleotide studies are highly sensitive but their specificity is too low, so that they are not practical in routine clinical practice $[13,14]$. Barium enema studies allow the diagnosis in $68-84 \%$ of all patients. They show a coil-spring appearance, indicating trapped barium between the inner and outer layer of the bowel loops [15]. Ultrasound of the short axis typically reveals two hypoechoic rings separated by a hyperechoic ring, the so called "doughnut sign" or "target sign". In the longitudinal plane, a pseudokidney structure or layering of hypoechoic lines with interspersed hyperechoic areas is observed. The sensitivity of ultrasound is examiner-dependent and reaches $98-100 \%$ with a specificity of $89 \%$ in children [16]. Superimposing air can result in inconclusive ultrasound examination [17, 18], and differentiation from bowel neoplasm can be impossible [10].

Fast magnetic resonance imaging (MRI) techniques reached good results in the detection of intussusception and achieved an accuracy rate of $96 \%$ compared to singleslice CT with $67-78 \%$ [7, 19]. Comparable data for MSCT do not exist. As the temporal resolution of MSCT is better than that of single-slice CT, abdominal image acquisition may be performed during breath hold, resulting to fewer respiration-related motion artifacts. Increased temporal resolution allows thinner collimation and enables the acquisition of high resolution volume data sets. As a result, interactive multiplanar reconstruction and volume rendering is readily performed and strongly facilitates diagnosis [18]. Colonoscopy has been recommended if the prevalent clinical symptoms indicate localization of intussusception in the colon [1]. This might be feasible if the clinical symptoms are mild, and colonoscopy is used as a supplementary examination to MSCT. Although successful colonoscopic reduction of intussusception has been reported [20], malignant etiology has to be excluded [21]. Aggressive work-up is not recommended in young patients with transient, short, non-obstructive enteroenteric intussusception $[4,22]$.

Table 1 List of lesions described in the literature causing intussusception in the small and large Intestine [1, 4, 7, 9, 10, 21, 24, 30, 31]

\begin{tabular}{lllll}
\hline Small intestine & & & Large intestine & Malignant \\
\cline { 5 - 5 } Benign & Malignant & Benign & Adenocarcinoma \\
\hline Adenom & Carcinomatosis & Adenomatous polyps & Carcinoid tumor \\
Celiac disease & Jejunal adenocarcinoma & Crohn disease & Cystadenocarcinoma \\
Cystic fibrosis & Leiomyosarcoma & & Cystic fibrosis & Kaposi sarcoma \\
Hamartomatous polyps & Metastatic melanoma & Endometriosis & Leiomyosarcom \\
Idiopathic & Metastatic sarcoma & Leiomyoma & Lymphoma \\
Local inflammation & Non-Hodgkin lymphoma & Mucoceles & Lymphosarcoma \\
Lymphoid hyperplasia & Undifferentiated carcinoma & & Previous anastomosis & Non-Hodgkin lymphoma \\
Meckel's diverticulum & & & & \\
Mesenteric papanniculitis & & & & \\
Neurofibroma & & & & \\
Postoperative scars & & & & \\
Scleroderma & & & & \\
Trauma & & & & \\
Zollinger Ellison syndrome & & &
\end{tabular}


Current imaging studies based on CT diagnosis differ from postoperative data. These studies showed that $70-84 \%$ of enteric and colocolic intussusceptions were not associated with any predisposing lesion [4, 23]. It has been suggested that these results represent a more physiological state based on functional abnormalities [23]. Results of previous studies might be biased by the selection of only postoperative patients, which might be of high risk for predisposing lesions [4]. CT allows as well the detection of transient intussusception reported in adults in the small intestine in combination with celiac disease [21, 24], Crohn's disease [25], or as an incidental finding [22], one case of transient colocolic intussusception has been described based on adenocarcinoma [26].

Intussusception occurs more frequently in the small $(50-88 \%)$ than in the large intestine $(12-50 \%)[1,4,7$, 9]. Benign lesions account for $50-64 \%$ of small intestinal intussusceptions and malignant lesions in 14-48\% [11]. Different types of benign lesions causing intussusception in the small intestine have been described (Table 1). Ninety-five percent of malignant lesions in the small intestine are metastatic. Only $1-5 \%$ are primary gastrointestinal neoplasias [7]. Idiopathic enteroenteric intussusception of the adult is rare $(2-23 \%)$, which is thought to be induced by diarrhea or by celiac or inflammatory diseases $[1,7]$. In the large bowel, idiopathic intussusception is even rarer than in the small intestine, with a frequency of $13 \%[1,3]$. Benign lesions of the large bowel account for about $30 \%$ of intussusceptions [10]. About $70 \%$ of intussusceptions of the large intestine have a malignant etiology [27] (Table 1). Areas exhibiting a high risk for adenoma exhibit a high risk for colon cancer and vice versa. About two thirds of the neoplastic lesions are adenocarcinoma, lymphoma [9], lymphosarcoma, and leiomyosarcoma $[3,4,10]$.

Appendiceal intussusception has been found together with benign lesions, including endometriosis, mucoceles, cystic fibrosis, Crohn's disease, fecaliths, worms, foreign bodies, and malignant lesions, including cystadenocarcinoma and carcinoid tumors $[1,28]$. The increased number of intussusceptions secondary to acquired immune deficiency syndrome (AIDS) is of growing interest. It is mostly ileal located and based on atypical mycobacterial infection, lymphoid hyperplasia, non-Hodgkin's lymphoma, or Kaposi's sarcoma [1, 29].

The extensive intussusception in the present patient demonstrated a target sign in sagittal MSCT reconstruction, with a concentric hyperdense double ring, corresponding to the different attenuation characteristics of mesenteric fat, mesenteric vessels, and the bowel wall of the intussusceptum and the intussuscipiens. Its length suggested an underlying lead lesion [4] detected at the tip of the intussusceptum.
Histopathology revealed a tubulovillous adenoma, which is relatively rare in patients below 40 years and more frequent in men $(61.6 \%)$ than in women $(38.4 \%)$ [27]. Their prevalence increases at the age of 40 years with a peak age of $60-70$ years. The high rate of underlying neoplastic lead lesions with a high prevalence of malignancy in adults in colocolic intussusception leads to the recommendation of en bloc resection without reduction as the primary operative treatment [1]. Resection has also been recommended in benign colonic lesions to prevent recurrent intussusception [7].

\section{Conclusion}

US, MRI, and single-slice CT showed high accuracy rates in the diagnosis of intussusception. MSCT allows the simultaneous assessment of the entire pathological process. Developments in CT technology, such as MSCT, allow volume data acquisition with high temporal and spatial resolution. Real-time multidimensional rendering techniques enable the independent diagnosis of the presence, location, and extension of intussusception in a one step examination. The detection of an intraluminal lead lesion is important. As patients with bowel obstruction are being increasingly investigated by $\mathrm{CT}$, clinician and radiologists will be confronted with this pathology more frequently. The CT features of intussusception are virtually pathognomonic and are easy to recognize. MSCT is accordingly the optimal method for pre-therapeutic diagnosis and for optimal treatment planning.

\section{References}

1. Begos DG, Sandor A, Modlin IM (1997) The diagnosis and management of adult intussusception. Am J Surg 173:88-94

2. Watson NA, Bisset RA (1994) Case report: intussusception-a cause of chronic abdominal symptoms and weight loss. Clin Radiol 49:723-726

3. Felix EL, Cohen MH, Bernstein AD, Schwartz JH (1976) Adult intussusception; case report of recurrent intussusception and review of the literature. Am J Surg 131:758-761

4. Warshauer DM, Lee JK (1999) Adult intussusception detected at CT or MR imaging: clinical-imaging correlation. Radiology 212:853-860

5. Agha FP (1986) Intussusception in adults. AJR Am J Roentgenol 146:527-531

6. Laws HL, Aldrete JS (1976) Small-bowel obstruction: a review of 465 cases. South Med J 69:733-734

7. Azar T, Berger DL (1997) Adult intussusception. Ann Surg 226:134-138

8. Wayne ER, Campbell JB, Burrington JD, Davis WS (1973) Management of 344 children with intussusception. Radiology 107:597-601

9. Nagorney DM, Sarr MG, McIlrath DC (1981) Surgical management of intussusception in the adult. Ann Surg 193:230-236 
10. Sato $M$, Ishida $H$, Konno $K$, Komatsuda $T$, Naganuma $H$, Watanabe S, Kotanagi H, Ishida J (2000) Long-standing painless intussusception in adults. Eur Radiol 10:811-813

11. Gayer G, Zissin R, Apter S, Papa M, Hertz M (2002) Pictorial review: adult intussusception-a CT diagnosis. Br J Radiol $75: 185-190$

12. Lande A, Schechter LS, Bole PV (1977) Angiographic diagnosis of small intestinal intussusception. Radiology 122:691-693

13. Kaminski CY, Kaminski JM, Guttentag AR, Tran H (2003) Positron emission tomography in intussusception of colon cancer, where computed tomography failed. Clin Nucl Med 28:60-61

14. Iko BO, Teal JS, Siram SM, Chinwuba CE, Roux VJ, Scott VF (1984) Computed tomography of adult colonic intussusception: clinical and experimental studies. AJR Am J Roentgenol 143:769-772

15. Sargent MA, Babyn P, Alton DJ (1994) Plain abdominal radiography in suspected intussusception: a reassessment. Pediatr Radiol 24:17-20

16. del Pozo G, Albillos JC, Tejedor D, Calero R, Rasero M, de la Calle U, Lopez-Pacheco U (1999) Intussusception in children: current concepts in diagnosis and enema reduction. Radiographics 19:299-319

17. Ledermann HP, Borner N, Strunk H, Bongartz G, Zollikofer C, Stuckmann G (2000) Bowel wall thickening on transabdominal sonography. AJR Am J Roentgenol 174:107-117

18. Mussack T, Szeimies U (2002) Sigmoidorectal intussusception caused by rectal carcinoma: multislice CT findings. Abdom Imaging 27:566-569

19. Beall DP, Fortman BJ, Lawler BC, Regan F (2002) Imaging bowel obstruction: a comparison between fast magnetic resonance imaging and helical computed tomography. Clin Radiol 57: $719-724$
20. Kitamura K, Kitagawa S, Mori M, Haraguchi Y (1990) Endoscopic correction of intussusception and removal of a colonic lipoma. Gastrointest Endosc 36:509-511

21. Gayer G, Apter S, Hofmann C, Nass S, Amitai M, Zissin R, Hertz M (1998) Intussusception in adults: CT diagnosis. Clin Radiol 53:53-57

22. Catalano O (1997) Transient small bowel intussusception: CT findings in adults. Br J Radiol 70:805-808

23. Lvoff N, Breiman RS, Coakley FV, Lu Y, Warren RS (2003) Distinguishing features of self-limiting adult small-bowel intussusception identified at CT. Radiology 227:68-72

24. Cohen MD, Lintott DJ (1978) Transient small bowel intussusception in adult coeliac disease. Clin Radiol 29:529-534

25. Knowles MC, Fishman EK, Kuhlman JE, Bayless TM (1989) Transient intussusception in Crohn disease: CT evaluation. Radiology 170:814

26. Zissin R, Lishner M, Rathaus V (1992) Case report: unusual presentation of splenic hamartoma; computed tomography and ultrasonic findings. Clin Radiol 45:410-411

27. Winawer SJ, Zauber AG, Ho MN, O'Brien MJ, Gottlieb LS, Sternberg SS, Stewart ET, Bond JH, Schapiro M, Panish JF (1993) The National Polyp Study. Eur J Cancer Prev 2(Suppl 2):83-87

28. Collins DC (1963) 71,000 human appendix specimens. A final report, summarizing forty years study. Am J Proctol 14:265-281

29. Visvanathan R, Nichols TT, Reznek RH (1997) Acquired immune deficiency syndrome-related intussusception in adults. Br J Surg $84: 1539-1540$

30. Naganuma H, Ishida H, Konno K, Komatsuda T, Sato M, Funaoka M, Fujimori S (1999) Intussusception in Peutz-Jeghers syndrome: sonographic findings. Abdom Imaging 24:333-335

31. Laredo J, Filtzer HS (2000) Right colonic intussusception. Am J Surg 179:485 